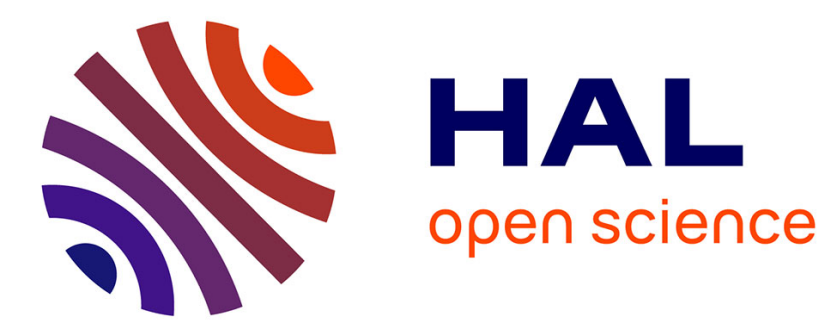

\title{
Evaporation en convection naturelle
}

\author{
M. Vachon, G. Arnaud, J.L. Peube
}

\section{To cite this version:}

M. Vachon, G. Arnaud, J.L. Peube. Evaporation en convection naturelle. Revue de Physique Appliquée, 1980, 15 (3), pp.427-431. 10.1051/rphysap:01980001503042700 . jpa-00244749

\section{HAL Id: jpa-00244749 https://hal.science/jpa-00244749}

Submitted on 1 Jan 1980

HAL is a multi-disciplinary open access archive for the deposit and dissemination of scientific research documents, whether they are published or not. The documents may come from teaching and research institutions in France or abroad, or from public or private research centers.
L'archive ouverte pluridisciplinaire HAL, est destinée au dépôt et à la diffusion de documents scientifiques de niveau recherche, publiés ou non, émanant des établissements d'enseignement et de recherche français ou étrangers, des laboratoires publics ou privés. 


\title{
Evaporation en convection naturelle
}

\author{
M. Vachon $(*)$, G. Arnaud (**) et J. L. Peube $\left({ }^{* *}\right)$ \\ ${ }^{*}$ ) Ingénieur Chercheur de l'Ecole Nationale des Ponts et Chaussées \\ $\left.{ }^{* *}\right)$ Laboratoire d'Energétique Solaire, BP 5, Odeillo, 66120 Font Romeu, France
}

(Reçu le 26 juillet 1979, révisé le 3 décembre 1979, accepté le 6 décembre 1979)

\begin{abstract}
Résumé. - On considère un écoulement laminaire permanent en convection naturelle engendré par des effets combinés de chaleur et de masse au voisinage d'une surface verticale humide chauffée par un flux constant $q$. Deux méthodes numériques sont présentées : la première, approchée, utilise les solutions affines qui décrivent les phénomènes en termes d'une variable de similitude et de quatre paramètres; la seconde est une méthode implicite de différences finies. Une comparaison des deux méthodes est présentée afin de tester les approximations des solutions affines.

Ces résultats théoriques sont ensuite comparés aux mesures expérimentales.
\end{abstract}

Abstract. - In this paper we present two numerical methods for solving stationary simultaneous heat and mass transfers in the laminar boundary layer on a vertical flat wet surface heated by constant heat flux. The first method develops equations in terms of a similarity variable and four dimensionless parameters. The second one uses an implicit finite difference procedure. A comparison of the two methods is presented.

A comparison between theoretical results and experimental measurements has been established.

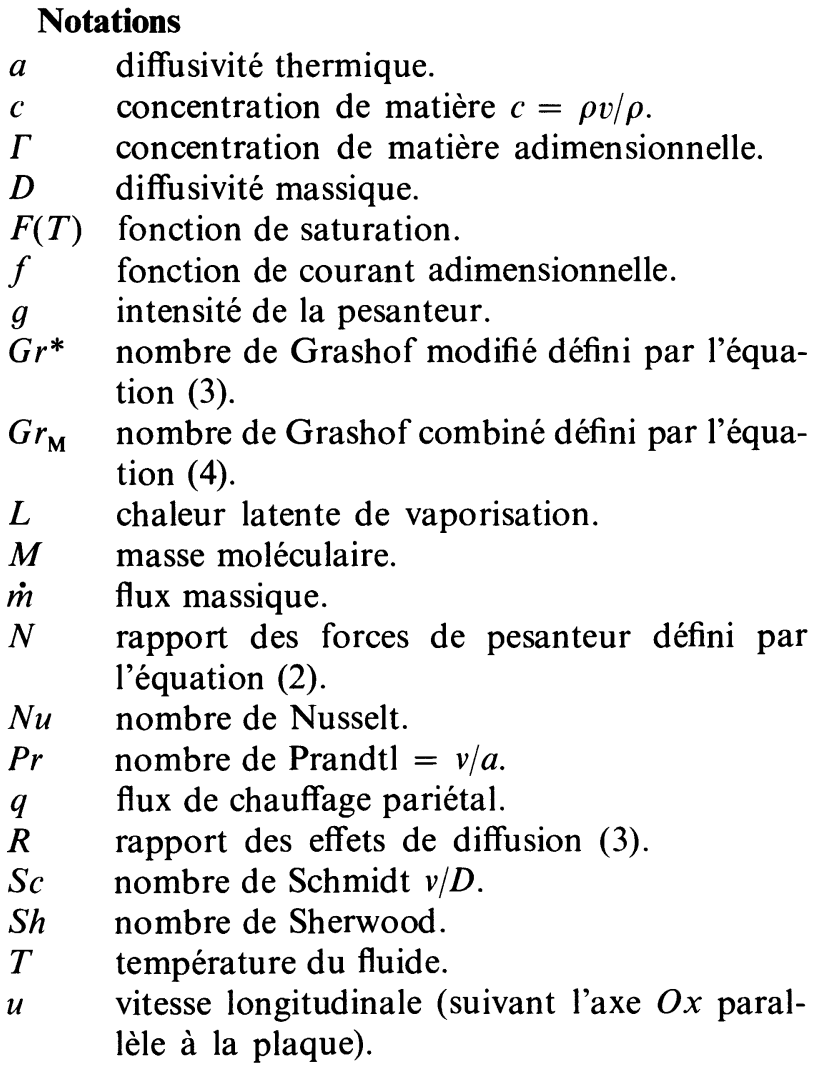

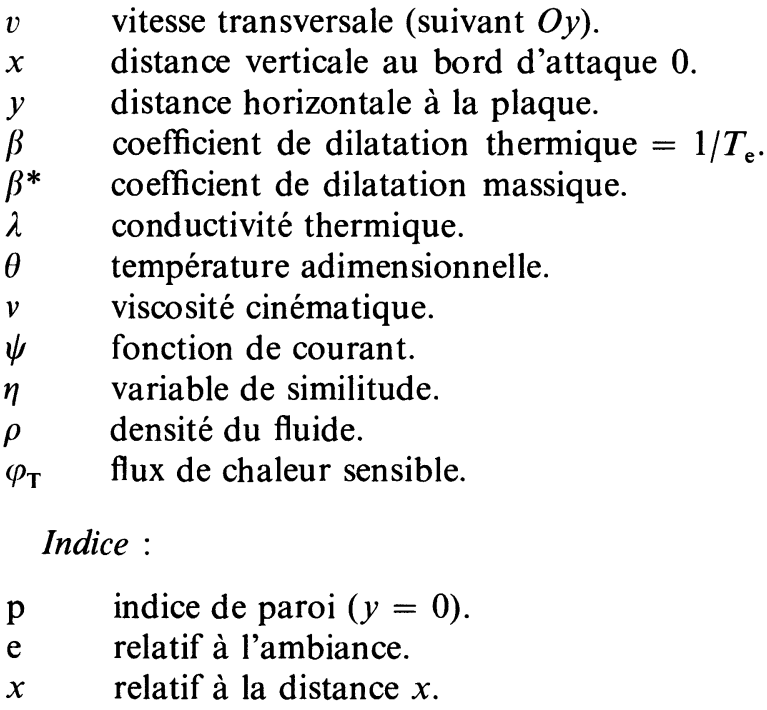

1. Introduction. - Les espaces tels que les serres, l'habitat sont caractérisés par' des transferts complexes de chaleur et de vapeur d'eau en convection naturelle. Cependant la convection naturelle en milieu confiné n'est pas tout à fait résolue et notamment la convection engendrée par des transferts simultanés de chaleur et de masse au voisinage de surfaces planes, 
verticales, chauffées par un flux constant n'a pas été étudiée. (Seul le cas du mur maintenu à température constante a été étudié $[1,2,3]$.$) La résolution de$ tels phénomènes est l'objet de notre travail qui contribue à l'établissement des bilans énergétiques dans les serres et l'habitat, et à la détermination des transferts liés aux corps humides chauffés par énergie solaire (évapotranspiration du couvert végétal).
2. Etude théorique. - 2.1 EQUATIONS GÉNÉRALES. - Les équations générales sont déduites des équations classiques de mécanique des fluides appliquées aux processus à faible gradient de matière, en supposant l'effet Soret et la diffusion thermique négligeables et en appliquant les hypothèses de Boussinesq ainsi que les hypothèses de couches limites laminaires bidimensionnelles :

$$
\left\{\begin{array}{l}
\frac{\partial u}{\partial x}+\frac{\partial v}{\partial y}=0 \\
u \frac{\partial u}{\partial x}+\imath \cdot \frac{\partial u}{\partial y}=v \frac{\partial^{2} u}{\partial y^{2}}+g \beta\left(T-T_{\mathrm{e}}\right)+g \beta^{*}\left(c-C_{\mathrm{e}}\right) \\
u \frac{\partial T}{\partial x}+\imath \frac{\partial T}{\partial y}=a \frac{\partial^{2} T}{\partial y^{2}} \\
u \frac{\partial c}{\partial x}+\imath \frac{\partial c}{\partial y}=D \frac{\partial^{2} c}{\partial y^{2}} \\
y=0 \quad u=v=0 ; \quad q=-\lambda\left(\frac{\partial T}{\partial y}\right)-L D \rho\left(\frac{\partial c}{\partial y}\right) ; \quad c(x, 0)=C_{\mathrm{p}}=F(T(x, 0)) \\
y \rightarrow \infty \quad u \rightarrow 0 \quad T \rightarrow T_{\mathrm{c}} \quad c \rightarrow C_{\mathrm{e}} .
\end{array}\right.
$$

Cette étude est restreinte au cas de l'évaporation de l'eau dans l'air $(\operatorname{Pr}=0,71, S c=0,63)$.

2.2 Solutions AFFines. - L'analyse [4] montre que le système (I) n'admet de solutions affines que lorsque la fonction de saturation est approchée par une fonction linéaire affine :

$$
F\left(T_{\mathrm{p}}\right)-C_{\mathrm{e}}=\alpha\left(T_{\mathrm{p}}-T_{\mathrm{e}}\right)=C_{\mathrm{p}}-C_{\mathrm{e}}
$$

soit, en terme adimensionnel, lorsque le rapport $N$ des effets d'Archimède est constant le long de la plaque :

$$
N=\frac{\beta^{*}\left(C_{\mathrm{p}}-C_{\mathrm{e}}\right)}{\beta\left(T_{\mathrm{p}}-T_{\mathrm{e}}\right)}=\frac{\alpha \beta^{*}}{\beta} .
$$

L'étude d'ordres de grandeur [4] montre que les écoulements ascendants $(u>0, x>0)$ sont caractérisés par des valeurs de $N$ telles que :

$$
\begin{array}{lll}
N>0 & \text { avec } & T_{\mathrm{p}}>T_{\mathrm{e}} \\
N<-1 & \text { avec } & T_{\mathrm{p}}<T_{\mathrm{e}} .
\end{array}
$$

Le domaine hachuré de la figure 1 indique le lieu de variation de la droite EN correspondant aux

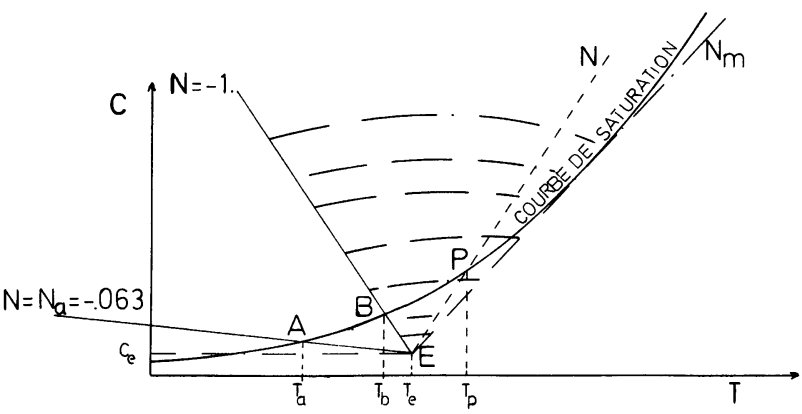

Fig. 1. - Schématisation du domaine de variation du rapport $N$. [Schematic variation for $N$ values.]

intervalles précédents. Cette représentation graphique montre que l'approximation de la courbe de saturation sera d'autant plus valable que le rapport $N$ sera proche de la valeur $\mathrm{Nm}$ correspondant à la tangente ENm.

Supposons cette approximation satisfaite. La fonction de courant $\psi$ et les différences de température et de concentration $\left(T-T_{\mathrm{e}}\right),\left(c-C_{\mathrm{e}}\right)$, sont écrites en fonction d'une variable $\eta$; soit $f, \theta, \Gamma$ les fonctions correspondantes :

$$
\begin{gathered}
\psi(x, y)=5 v \mathrm{~d}(x) f(\eta) ; \quad 0(\eta)=\frac{\lambda\left(T-T_{\mathrm{e}}\right)}{q x} \mathrm{~d}(x) ; \quad \Gamma(\eta)=\frac{L D \rho\left(c-C_{\mathrm{e}}\right)}{R q \cdot x} \mathrm{~d}(x) \\
\eta=\frac{y}{x} \mathrm{~d}(x) ; \quad R=\frac{L D \rho \beta}{\lambda \beta^{*}} ; \quad \mathrm{d}(x)=\sqrt[5]{\frac{g \beta q x^{4}}{5 \lambda v^{2}}}=\sqrt[5]{\frac{G r^{*} x}{5}} .
\end{gathered}
$$


Le système aux dérivées partielles (I) se transforme en un système différentiel (II) :

$$
\begin{gathered}
f^{\prime \prime \prime}-3 f^{2}+4 f^{\prime \prime} f+\Gamma+\theta=0 \\
0^{\prime \prime}+\operatorname{Pr}\left(4 f \theta^{\prime}-f^{\prime} \theta\right)=0 \\
\Gamma^{\prime \prime}+S c\left(4 f \Gamma^{\prime}-f^{\prime} \Gamma\right)=0 \\
\eta=0:-1=\theta^{\prime}(0)+R \Gamma^{\prime}(0) ; \quad \Gamma(0)=N \theta(0) ; \quad f(0)=f^{\prime}(0)=0 \\
\eta \rightarrow \infty \quad f^{\prime} \rightarrow 0 \quad 0 \rightarrow 0 \quad \Gamma \rightarrow 0 .
\end{gathered}
$$

La résolution numérique du système (II) a été réalisée par une méthode de Runge Kutta Gill et une méthode de tir corrigé permettant d'évaluer les trois valeurs inconnues $f^{\prime \prime}(0), \theta(0), \theta^{\prime}(0)$ [4]. La figure 2 rassemble quelques courbes de variation de température $\theta$ et de vitesse adimensionnelle $f^{\prime}$ en fonction de la variable $\eta$ et du paramètre $N$.

Pour représenter les transferts de chaleur et de masse, on introduit les nombres de Nusselt et de Sherwood, et on constate qu'en utilisant un autre nombre de Grashof $G r_{\mathrm{M}}$, les relations de transferts se simplifient et sont données numériquement par les formules suivantes :

avec

$$
\begin{aligned}
& N u_{x}=\frac{\varphi_{\mathrm{T}} x}{\lambda\left(T_{\mathrm{p}}-T_{\mathrm{e}}\right)}=0,49\left(G r_{\mathrm{M}}\right)^{1 / 5} \pm 1 \% \\
& S h_{x}=\frac{\dot{m} x}{D_{\rho}\left(C_{\mathrm{p}}-C_{\mathrm{e}}\right)}=0,462\left(G r_{\mathrm{M}}\right)^{1 / 5} \pm 1 \%
\end{aligned}
$$

$$
\begin{gathered}
q=\rho_{\mathrm{T}}+L \dot{m} \\
G r_{\mathrm{M}}=\frac{g \beta \rho_{\mathrm{T}} x^{4}}{\lambda v^{2}}+\frac{g \beta^{*} \dot{m} x^{4}}{D_{\rho} v^{2}}=-\left(\theta^{\prime}(0)+\Gamma^{\prime}(0)\right) G r_{x}^{*}=p(N) G r_{x}^{*} .
\end{gathered}
$$

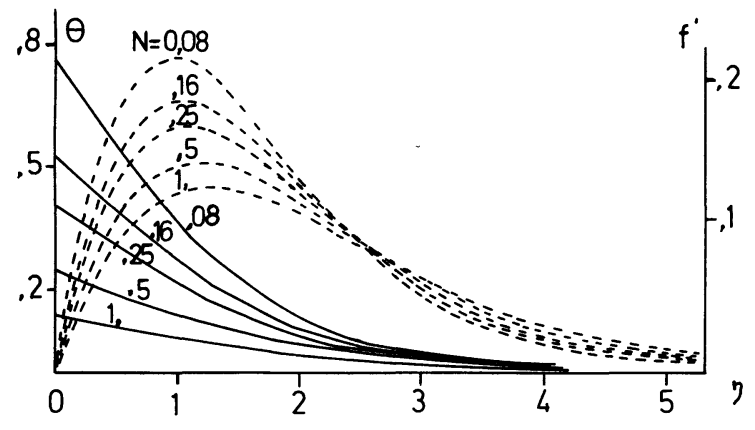

Fig. 2. - Profils adimensionnels 0 et $f^{\prime}$ en fonction de la variable de similitude $\eta$ et du rapport $N .(\operatorname{Pr}=0,71, S c=0,63, R=16,8$.

[Dimensionless profiles $\theta$ and $f^{\prime}$ in function of the similarity variable $\eta$ and the parameter $N .(\operatorname{Pr}=0.71: S c=0.63: R=16.8)$.

2.3 CAS D'UNE FONCTION DE SATURATION QUELCONQUE. - Le rapport $N$ n'est plus constant le long de la plaque. Nous avons donc mis au point une méthode approchée qui admet la validité des solutions précédentes et qui détermine par itération le rapport $N$ pour chaque abscisse $x$ et pour des conditions aux limites $\left(T_{\mathrm{e}}, C_{\mathrm{e}}, q\right)$ quelconques : une valeur $N_{0}$ est prise arbitrairement; les solutions affines conduisent à une valeur $\theta(0)$ donc à une température de paroi :

$$
T_{\mathrm{p}}=\frac{\theta(0) q x}{\lambda \mathrm{d}(x)}+T_{\overline{\mathrm{e}}} .
$$

La connaissance de $T_{\mathrm{p}}(x)$ permet de calculer une concentration pariétale $C_{\mathrm{p}}=F\left(T_{\mathrm{p}}\right)$ et une nouvelle valeur de $N$. Si les deux valeurs $N$ et $N_{0}$ sont proches, la valeur de $N$ est atteinte sinon on poursuit l'itération. Ce calcul permet d'évaluer rapidement un rapport $N$ approché pour un quadruplet quelconque $\left(x, T_{\mathrm{e}}, C_{\mathrm{e}}, q\right)$.

Ces calculs montrent que plus le flux $q$ est élevé, plus la condition $N=$ constant se réalise rapidement (à partir de $\left.x_{0} \sim 0,1\right)$ et que, à flux donné, cette condition se réalise d'autant mieux que la température ambiante est basse ou que l'humidité ambiante est élevée (Fig. 3). Notons $x_{0}$, abscisse de stabilisation (i.e. $x>x_{0}$, variation de $N$ inférieure à $20 \%$ ).

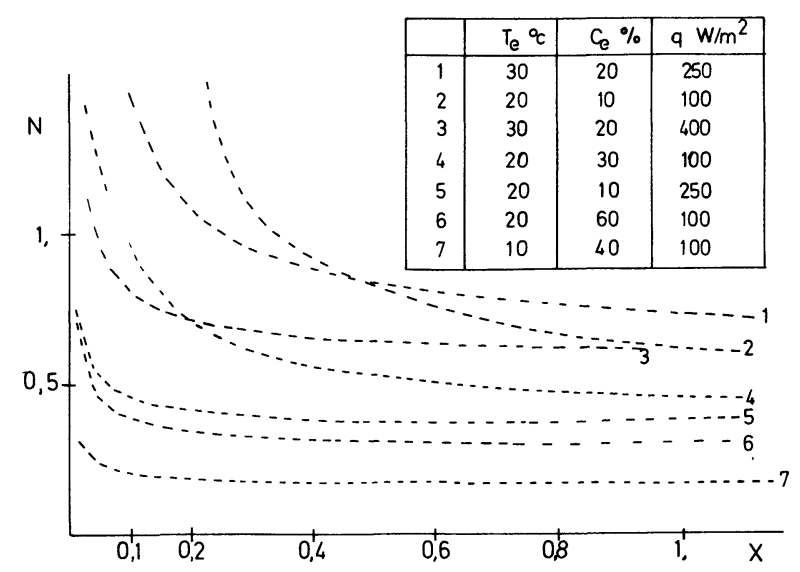

Fig. 3. - Détermination approchée du rapport $N$ en fonction de la hauteur $x$ et des conditions aux limites $\left(T_{\mathrm{e}}, C_{\mathrm{e}}, q\right)$.

[Profiles $N$ in function of the vertical distance $x$ and of the boundary conditions $\left(T_{\mathrm{e}}, C_{\mathrm{e}}, q\right)$.] 
2.4 VALIDATION DE LA MÉTHODE APPROCHÉE. Les résultats précédents montrent que l'hypothèse $N=$ constant n'est réalisée qu'à partir d'une certaine abscisse $x_{0}$. Or, un écoulement a son histoire, comparable à un événement, et toute perturbation de cette histoire peut se répercuter sur son avenir. C'est pourquoi, nous avons mis au point une méthode implicite de différences finies, afin de tester la validité des approximations précédentes. Cette procédure ne fait aucune supposition sur la fonction de saturation, cependant son exploitation numérique nécessite une expression analytique de cette fonction. En particulier, nous avons utilisé une approximation de la pression de vapeur saturante $p_{\mathrm{vs}}$ du type :

$$
\begin{array}{rl}
\log p_{\mathrm{vs}}(T)=28,590 & 51-8,2 \log T \\
+ & 2,4804 \times 10^{-3} T-3142,32 / T
\end{array}
$$

la pression de vapeur étant exprimée en atmosphère et $T$ en degré kelvin.

Après avoir défini un maillage du domaine d'intégration, $[(x, y)=j \Delta x, n \Delta y]$, le système (I) discrétisé, linéarisé, se transforme en équations matricielles :

$$
j \rightarrow \cup \quad L_{j} W_{j+1}=D_{j}
$$

les conditions initiales, associées à la ligne " $j=0$ » correspondent au bord d'attaque $0(x=0)$. L'évaluation des variables de vitesse, de température et d'humidité en ce point, est assez complexe, d'autant que l'écoulement y subit une discontinuité et que les équations de couches limites ne sont plus applicables. Nous avons contourné le problème en assimilant les conditions initiales aux conditions ambiantes $\left(T(0, y)=T_{\mathrm{e}} ; c(0, y)=C_{\mathrm{e}} ; u(0, y)=v(0, y)=0\right)$.

Les matrices $L_{j}$ étant tridiagonales par bloc, les équations matricielles (III) ont été résolues par une méthode d'élimination de Gauss pour différentes conditions aux limites [4].

Les diverses résolutions montrent que les évaluations du rapport $N$ par les deux méthodes numériques coïncident à $2 \%$ près à partir d'une abscisse $x_{1}$ ( $x_{1}$ est de l'ordre de 1 à $10 \mathrm{~cm}$ ). Cette imprécision au niveau du bord d'attaque est certainement due aux fortes variations inhérentes à ce point particulier et aussi à la difficulté de bien saisir les phénomènes à ce niveau de la plaque. Cependant, les perturbations liées au bord d'attaque ont un faible rayon d'action et ne se répercutent pas sur l'ensemble de la plaque.

De plus la comparaison des divers profils obtenus par les deux méthodes numériques, montre des écarts de 8 à $15 \%$ avant d'atteindre l'abscisse de stabilisation $x_{0}$. A partir de cette abscisse $x_{0}$, ces écarts passent progressivement à $2-5 \%$ (cf. Fig. 4). Le programme de différences finies a tendance à diminuer l'épaisseur de la couche limite dynamique, de sorte que les écarts dans la région externe de l'écoulement sont plus importants.

Par conséquent, la méthode approchée donne une bonne approximation de la solution générale à partir

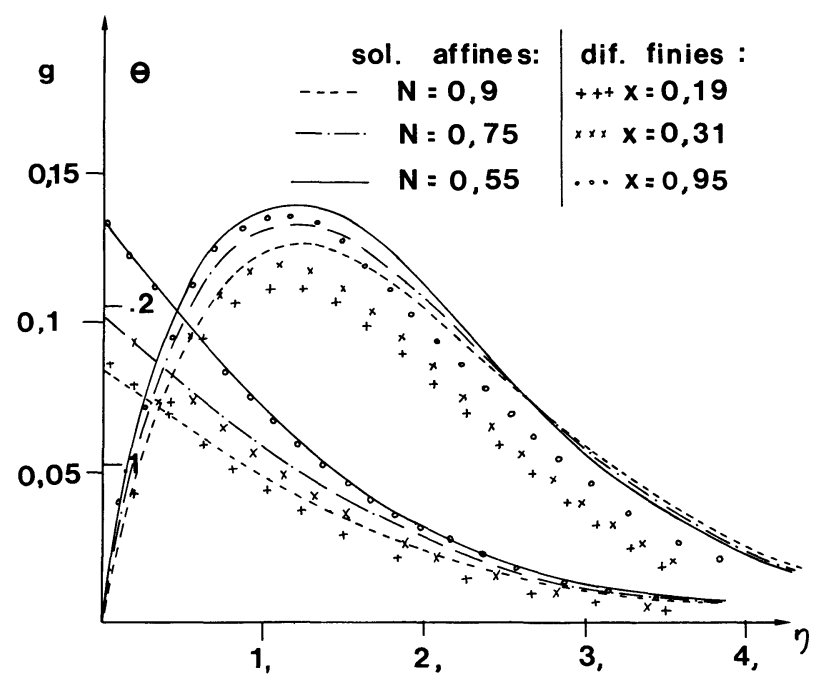

Fig. 4. - Comparaison solutions affines - différences finies $\left(T_{\mathrm{e}}=20^{\circ} ; C_{\mathrm{e}}^{*}=30 \% ; q=100 \mathrm{~W} / \mathrm{m}^{2}\right)$.

[Comparison between similarity solutions and finite differences procedure $\left(T_{\mathrm{e}}=20^{\circ}: C_{\mathrm{e}}^{*}=30 \%: q=100 \mathrm{~W} / \mathrm{m}^{2}\right)$.]

de l'abscisse de stabilisation $x_{0}$; elle peut donc se substituer à la méthode des différences finies d'autant mieux que cette abscisse est faible.

3. Etude expérimentale. - Parallèlement à l'étude théorique, un modèle expérimental a été réalisé (Fig. 5). Il se compose d'une plaque poreuse en alumine électrofondue de $1 \mathrm{~m}$ de haut, $0,30 \mathrm{~m}$ de large. Cette plaque est humidifiée par gravité, chauffée par un système de 12 lampes infrarouges assurant à $5 \%$ près l'uniformité du flux.

Ce panneau chauffant fournit un flux de $450 \mathrm{~W} / \mathrm{m}^{2}$ à $1 \mathrm{~m}$ de la plaque. Les mesures de vitesses et de températures ont été réalisées à l'aide d'une sonde anémométrique vibrante dont le déplacement est commandé à distance pour éviter toutes perturbations et se rapprocher des conditions optimales de mesure en convection naturelle. Ce déplacement permet de prospecter la couche limite à différentes hauteurs de plaque. Aucune mesure d'humidité dans

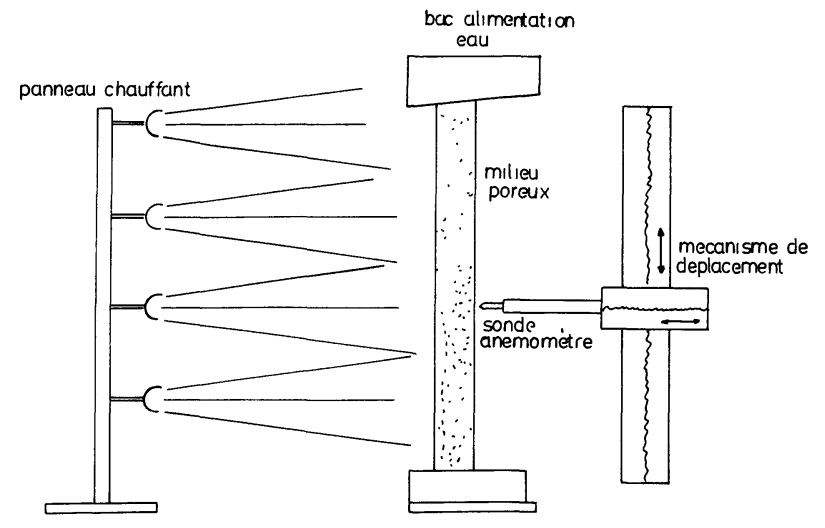

Fig. 5. - Schéma du montage expérimental.

[Schematic drawing of experimental unit.] 
la couche limite n'a été faite étant donné les difficultés de telles mesures. Seule l'humidité ambiante ainsi que la température ambiante ont été relevées sur un hygrothermoenregistreur étalonné sur un psychromètre.

Les figures 6 et 7 donnent quelques exemples de comparaison entre les résultats théoriques et expérimentaux.

Ces profils témoignent d'un accord satisfaisant entre les deux modèles notamment dans la région interne de l'écoulement. Dans la région externe, les écarts sont plus importants : ceci est dû à la difficulté de maintenir des conditions rigoureuses de convection naturelle.

Etant donné la précision de la sonde anémométrique, nous n'avons pas pu réaliser des mesures près du bord d'attaque, et ainsi comparer les résultats théoriques et expérimentaux dans les zones de fortes variations du rapport $N\left(x<x_{0}\right)$.

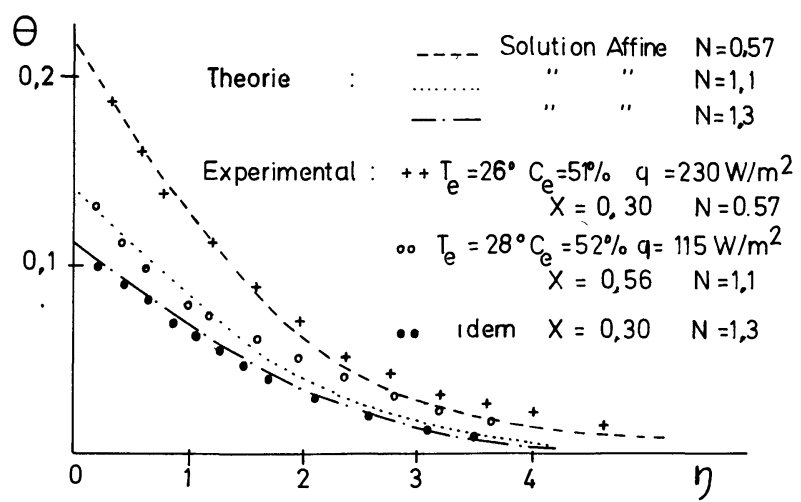

Fig. 6. - Comparaison des profils de températures adimensionnelles $\theta$ théoriques et expérimentaux

[Comparison between theoretical and expermental temperature profiles.]

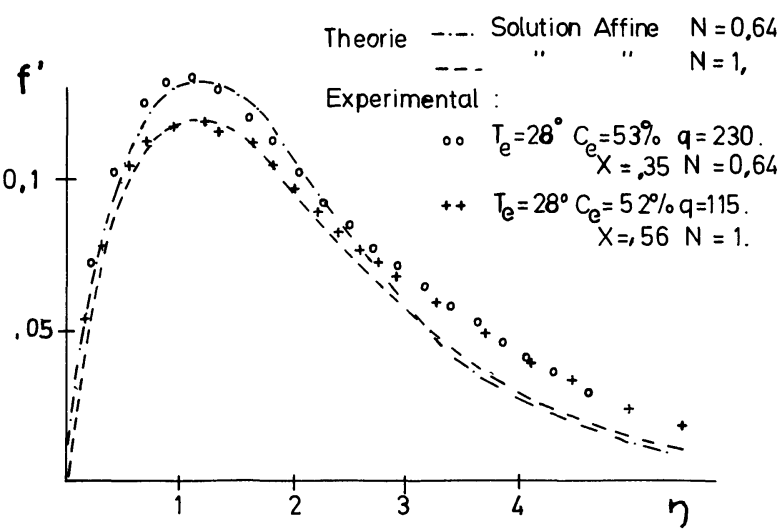

Fig. 7. - Comparaison des profils de vitesses adimensionnelles $f^{\prime}$ théoriques et expérimentaux.

[Comparison between theoretical and experimental velocity profiles.]

4. Conclusion. - Ce présent rapport contribue à l'étude des transferts simultanés de chaleur et de vapeur d'eau le long de surfaces humides soumises par un flux pariétal constant.

La méthode approchée des solutions affines permet dans la majorité des cas d'interpréter de tels phénomènes. Cette étude montre l'influence du rapport $N$ des effets d'Archimède et fait apparaître un nombre de Grashof combiné qui prend en compte les deux variétés de flux (flux de chaleur latente et flux de chaleur sensible).

L'intérêt de tels résultats est de fournir des solutions approchées et des relations de transferts concernant l'évolution de la couche limite dynamique, thermique et massique et de permettre de résoudre simplement les problèmes d'évaporation en convection naturelle.

\section{Bibliographie}

[1] Gebhart, B., Pera, L., Int. J. Heat Mass Trans. 14 (1979) 2025-2050

[2] Gai i ahan. G. D.. Warnfr. W. J.. Int. J. Hecat Mass Trans. 19 (1976) 165-174.
[3] Bottemane, F. A., Appl. Sci. Res. 25 (1971) 137-149.

[4] VACHON, M., Thèse docteur ingénieur soutenue en déc. 1979 Université de Poitiers. 